\title{
Pyloric Stenosis in a Patient with Systemic Lupus Erythematosus
}

A 38-year-old Japanese woman, who had fulfilled the revised American College of Rheumatology criteria for systemic lupus erythematosus (SLE), and whose disease had been controlled with oral prednisolone at a dose of $5 \mathrm{mg} /$ day for a year, reported a 3-day history of nausea and vomiting. On admission, laboratory tests showed a lactate dehydrogenase level of $610 \mathrm{IU} / \mathrm{l}$, an erythrocyte sedimentation rate of $35 \mathrm{~mm}$ /hour, a positive antinuclear antibody (titer 1:40), and decreased levels of complements (C3, $32 \mathrm{mg} / \mathrm{dl}$; $\mathrm{C} 4$, $6 \mathrm{mg} / \mathrm{dl}$; CH50, 24.4 U/dl). Gastroscopic examination revealed a stenosis of the pyloric antrum with no mucosal lesion (Figure 1). A computed tomographic scan demonstrated diffuse thickening of the antral wall with marked enhancement by contrast material on the serosal side (Figure 2), in addition to mild hydronephrosis, thickened wall of the gallbladder, and a small amount of ascites. The lupus peritonitis and cholecystitis was thought to be the most likely cause of the antral stenosis.

The patient was treated with intravenous prednisolone at a dose of $20 \mathrm{mg} /$ day and the symptoms subsided immediately. Endoscopic examination and a computed tomographic scan within 2 weeks after the introduction of intravenous steroids showed no evidence of antral wall thickening or stenosis. The patient has been
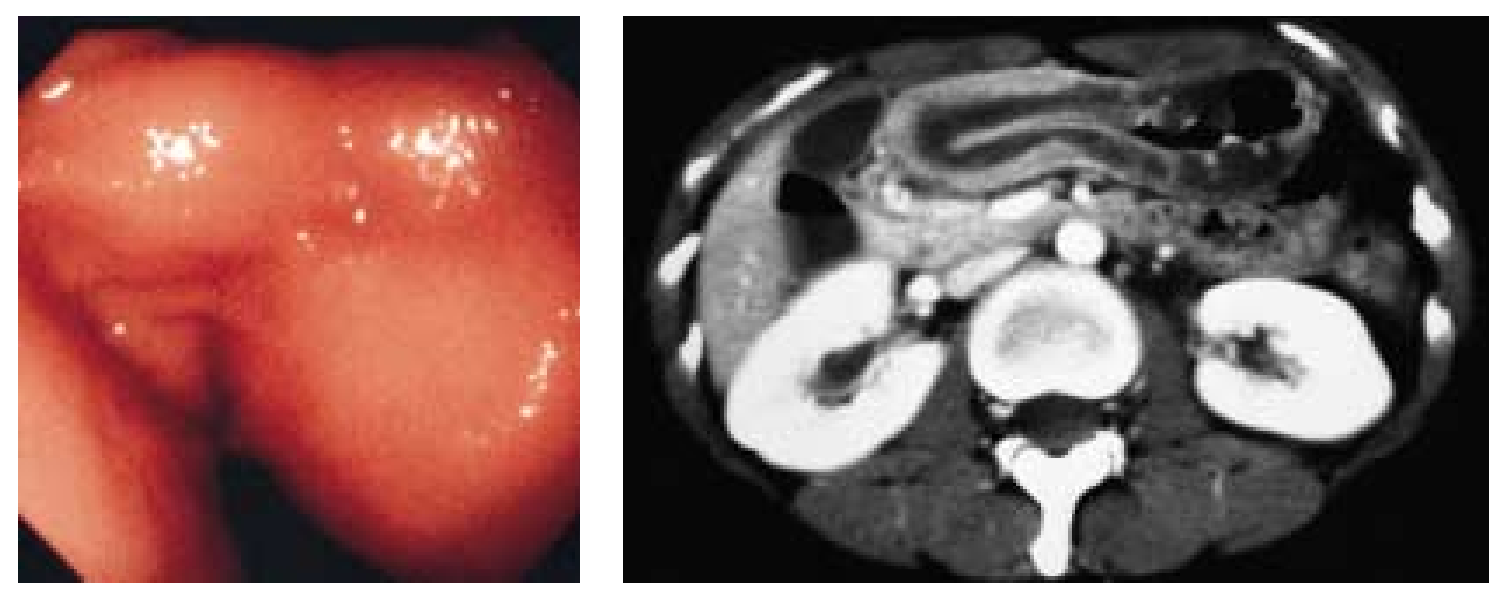

free from symptoms over 1 year of follow-up.

Lupus peritonitis and cystitis is an unusual manifestation of SLE. In previously reported cases, involvement of the gut has been primarily in the small intestine [1-3] and rarely in the stomach $[4,5]$. The finding in our patient indicates that serositis can occur focally on the gastric serosa in SLE, as shown previously at laparotomy [5], which results in stenosis of the pyloric antrum. A contrast computed tomography (CT) scan and endoscopic examination are useful for the differential diagnosis. Physicians should consider this unusual cause of antral stenosis which showed adequate remission with intravenous steroid.

\section{H. Takano ${ }^{1}$, K. Inoue ${ }^{1}$, K. Katada ${ }^{2}$, T. Yoshikawa ${ }^{2}$}

${ }^{1}$ National Institute for Environmental Studies, Tsukuba, Japan

${ }^{2}$ Kyoto Prefectural University of Medicine, Kyoto, Japan

\section{References}

${ }^{1}$ Weisman MH, McDonald EC, Wilson CB. Studies of the pathogenesis of interstitial cystitis, obstructive uropathy, and intestinal malabsorption in a patient with systemic lupus erythematosus. Am J Med 1981; 70: 875-881
${ }^{2}$ Vicencio GP, Chung-Park M, Ricanati E et al. SLE with interstitial cystitis, reversible hydronephrosis and intestinal manifestations. J Rheumatol 1989; 16: 250-251

${ }^{3}$ Moriuchi J, Ichikawa Y, Takaya M et al. Lupus cystitis and perforation of the small bowel with a systemic lupus erythematosus and overlapping syndrome. Clin Exp Rheumatol 1989; 7: 533-536

${ }^{4}$ Posthuma EF, Warmerdam P, Chandie Shaw MP et al. Gastric outlet obstruction as a presenting manifestation of systemic lupus erythematosus. Gut 1994; 35: $841-843$

${ }^{5}$ Case Records of the Massachusetts General Hospital. N Engl J Med 1959; 260: 1035-1039

\section{Corresponding Author}

\section{H. Takano, M.D., Ph.D.}

National Institute

for Environmental Studies

16-2 Onogawa

Tsukuba 305-0053

Japan

Fax: $\quad+81-298-502334$

E-mail: htakano@nies.go.jp avesalius@padova.uni.gov.it
Figure 1 Gastroscopic examination showed stenosis of the pyloric antrum with no mucosal lesion.
Figure 2 A computed tomography (CT) scan of the abdomen demonstrated diffuse thickening with marked enhancement by contrast material on the serosal side. of the antral wall 